\title{
Provider confidence in the telemedicine spine evaluation: results from a global study
}

\author{
Francis Lovecchio ${ }^{1}$ - Grant J. Riew ${ }^{2}$ - Dino Samartzis ${ }^{3,4}$. Philip K. Louie ${ }^{5} \cdot$ Niccole Germscheid $^{6} \cdot$ Howard S. An $^{3,4}$. \\ Jason Pui Yin Cheung ${ }^{7}$. Norman Chutkan ${ }^{8}$. Gary Michael Mallow ${ }^{3,4}$ • Marko H. Neva ${ }^{9}$. Frank M. Phillips ${ }^{3,4}$. \\ Daniel M. Sciubba ${ }^{10} \cdot$ Mohammad El-Sharkawi $^{11}$ - Marcelo Valacco ${ }^{12} \cdot$ Michael H. McCarthy ${ }^{13} \cdot$ Melvin C. Makhni $^{2}$. \\ Sravisht lyer ${ }^{1}$
}

Received: 22 September 2020 / Accepted: 27 October 2020 / Published online: 22 November 2020

(c) The Author(s) 2020

\begin{abstract}
Purpose To utilize data from a global spine surgeon survey to elucidate (1) overall confidence in the telemedicine evaluation and (2) determinants of provider confidence.

Methods Members of AO Spine International were sent a survey encompassing participant's experience with, perception of, and comparison of telemedicine to in-person visits. The survey was designed through a Delphi approach, with four rounds of question review by the multi-disciplinary authors. Data were stratified by provider age, experience, telemedicine platform, trust in telemedicine, and specialty.

Results Four hundred and eighty-five surgeons participated in the survey. The global effort included respondents from Africa (19.9\%), Asia Pacific (19.7\%), Europe (24.3\%), North America (9.4\%), and South America (26.6\%). Providers felt that physical exam-based tasks (e.g., provocative testing, assessing neurologic deficits/myelopathy, etc.) were inferior to inperson exams, while communication-based aspects (e.g., history taking, imaging review, etc.) were equivalent. Participants who performed greater than 50 visits were more likely to believe telemedicine was at least equivalent to in-person visits in the ability to make an accurate diagnosis (OR 2.37, 95\% C.I. 1.03-5.43). Compared to in-person encounters, video (versus phone only) visits were associated with increased confidence in the ability of telemedicine to formulate and communicate a treatment plan (OR 3.88, 95\% C.I. 1.71-8.84).

Conclusion Spine surgeons are confident in the ability of telemedicine to communicate with patients, but are concerned about its capacity to accurately make physical exam-based diagnoses. Future research should concentrate on standardizing the remote examination and the development of appropriate use criteria in order to increase provider confidence in telemedicine technology.
\end{abstract}

Keywords Telemedicine $\cdot$ Spine surgery $\cdot$ Examination $\cdot$ International $\cdot$ Survey

Sravisht Iyer

iyers@hss.edu

1 Department of Orthopaedic Surgery, Hospital for Special Surgery, New York, NY, USA

2 Department of Orthopaedic Surgery, Brigham and Women's Hospital, Harvard Medical School, Boston, MA, USA

3 Department of Orthopaedic Surgery, Rush University Medical Center, Chicago, IL, USA

4 The International Spine Research and Innovation Initiative, Rush University Medical Center, Chicago, IL, USA

5 Neuroscience Institute, Virginia Mason Medical Center, Seattle, WA, USA

6 Research Department, AO Spine International, Davos, Switzerland
7 Department of Orthopaedics \& Traumatology, The University of Hong Kong, Hong Kong SAR, China

8 Department of Orthopaedic Surgery, University of Arizona College of Medicine, Phoenix, AZ, USA

9 Department of Orthopaedic and Trauma Surgery, Tampere University Hospital, Tampere, Finland

10 Department of Neurosurgery, Baltimore, MD, USA, John Hopkins University, Baltimore, MD, USA

11 Department of Orthopaedic and Trauma Surgery, Assiut University Medical School, Assiut, Egypt

12 Department of Orthopaedics, Churruca Hospital de Buenos Aires, Buenos Aires, Argentina

13 Indiana Spine Group, Carmel, IN, USA 


\section{Introduction}

Though COVID-19 accelerated the worldwide adoption of remote health care, recent trends had already established telemedicine as a fast-growing clinical tool [1]. The last two decades have witnessed the expansion of telemedicine in general surgery, medical education, and surgical subspecialties [2, 3], a trend now cemented in place by current COVID-19 restrictions. Spine surgery has also experienced similar growth and adoption of this practice change [4].

Certain challenges are inevitable in the widespread adoption of any "new" mode of care delivery, especially with the incorporation of new technology. Barriers to the adoption of telemedicine in spine surgery include the implementation of novel technology, lack of technological literacy, new medicolegal territory, negative financial implications, and regulatory concerns [5]. However, as with any new technology, the largest barrier to widespread adoption is user confidence in the clinical utility of telemedicine. Whether telemedicine will play a large or small part in the overall patient evaluation remains to be seen. One controversial aspect of telemedicine is the ability to perform accurate physical examination, as experts diverge on how best to examine patients and how much faith can be placed in these virtual evaluations [6-8].

Understanding surgeon confidence in telemedicine will be necessary to develop appropriate use criteria, improve deficient areas, and standardize the telemedicine evaluation. International perspectives are particularly valuable as they offer the widest array of experience and opinion. The purpose of this study was to utilize data from a global spine surgeon survey to elucidate (1) overall confidence in the telemedicine evaluation and (2) determinants of provider confidence in this novel method of patient evaluation.

\section{Methods}

\section{Survey design}

The data utilized in this study were retrieved from a crosssectional, global survey designed to capture spine surgeons' perspectives on telemedicine. The survey was designed through a Delphi approach, in which all questions underwent four rounds of review by the multi-disciplinary study authors [9]. The final survey consisted of 42 questions encompassing seven major categories: demographics, usage of telemedicine, patient perception, trust in telemedicine, challenges and benefits, comparison to in-person visits, and training and research (Appendix 1). The survey was anonymous, as no identifying information was collected from respondents.

\section{Study sample}

The survey entitled "Telemedicine $\&$ the Spine SurgeonPerspectives and Practices Worldwide" was distributed through email to members of AO Spine starting May 15, 2020, with closure of the survey on May 31, 2020. AO Spine is the largest international society dedicated to spine surgery, consisting of over 30,000 professionals, of which greater than 6000 are practicing spine surgeons (www.aospine.org). The survey was distributed to the 3805 surgeons who opted in to receive email surveys.

\section{Statistical analyses}

Survey respondents were grouped into five geographical regions: Africa, Asia-Pacific, Europe, North America, and South America. All questions were considered optional; thus, pairwise deletion was utilized for missing data points. In order to present the large content of the survey in a concise manner, statistical analyses were divided into four major themes: global perspectives, challenges and benefits, telemedicine evaluation, and training and research. Thus, the current analysis focuses specifically on survey questions pertaining to the telemedicine evaluation.

The data were stratified by region, provider age, telemedicine platform, experience with telemedicine, specialty, and trust in telemedicine, and compared. To determine participants' sense of trust in telemedicine, the survey query: "If you or a family member were a patient, do you believe the initial visit can be performed through telemedicine?" was utilized as an anchor question, with a response of "agree" or "strongly agree" classified as a positive response. Likert scale questions were analyzed as both categorical and continuous variables. Categorical variables were compared using Pearson's Chi-square test and continuous variables were compared using Mann-Whitney U tests or ANOVA test as appropriate. To control for confounding and isolate factors independently associated with confidence in the telemedicine evaluation, multivariate logistic regressions were performed for each survey question. Odds ratios (OR) and 95\% confidence intervals (CI) were assessed for precision. Hosmer-Lemeshow tests were used to ensure appropriate goodness of fit for all models. All statistical analyses were performed using SPSS version 25.0 (IBM, Armonk, NY), and the threshold for statistical significance was established at $p<0.05$. 


\section{Results}

\section{Survey sample}

In total, 485 spine surgeons participated in the survey. Most survey participants were between 35-44 (68.7\%) and 45-54 (33.0\%) years old, and the vast majority were male (94.5\%). Responses were split between Africa (19.9\%), Asia Pacific (19.7\%), Europe (24.3\%), North America (9.4\%), and South America (26.6\%). Videoconferencing platforms (EMR-integrated or non-secure) were utilized by $57.5 \%$, while $34.6 \%$ of respondents used telephone calls only for telemedicine. At the time of the survey, most participants had performed fewer than 50 visits (77.8\%), with less than a quarter of having performed over 50 telehealth visits $(22.2 \%)$ (Table 1).

\section{Confidence in the telemedicine evaluation-overall responses}

Participants were asked to compare telemedicine to in-person visits among multiple facets of the patient evaluation (i.e., history taking, examination, imaging review, diagnosis). Analysis of the mean Likert scale responses demonstrated an overall leftward skew of provider opinion toward telemedicine evaluation (Fig. 1, Table 2). Most respondents felt that telemedicine was at least equivalent to in-person visits for communicative tasks, such as taking a patient history (64.3\%),

Table 1 Survey respondent demographics

\begin{tabular}{|c|c|c|c|c|c|}
\hline & $n$ & Percent $^{\mathrm{a}}$ & & $n$ & Percent \\
\hline Sex & & & Specialty & & \\
\hline Male & 446 & $94.5 \%$ & Orthopedics & 332 & $68.5 \%$ \\
\hline Female & 26 & $5.5 \%$ & Neurosurgery & 144 & $29.7 \%$ \\
\hline Age (years) & & & Trauma & 50 & $10.3 \%$ \\
\hline $25-34$ & 56 & $11.7 \%$ & Pediatric surgery & 16 & $3.3 \%$ \\
\hline $35-44$ & 173 & $36.1 \%$ & Other & 14 & $2.9 \%$ \\
\hline $45-54$ & 160 & $33.4 \%$ & Years practicing spine surgery & & \\
\hline $55-64$ & 73 & $15.2 \%$ & $0-5$ & 100 & $20.9 \%$ \\
\hline $65+$ & 17 & $3.5 \%$ & $5-10$ & 116 & $24.3 \%$ \\
\hline Geographic region & & & $11-15$ & 82 & $17.2 \%$ \\
\hline Africa & 95 & $19.9 \%$ & $16-20$ & 68 & $14.2 \%$ \\
\hline Asia Pacific & 94 & $19.7 \%$ & $20+$ & 112 & $23.4 \%$ \\
\hline Europe & 116 & $24.3 \%$ & Telemedicine platform & & \\
\hline North America & 45 & $9.4 \%$ & Phone & 100 & $34.6 \%$ \\
\hline South America & 127 & $26.6 \%$ & Video & 116 & $57.5 \%$ \\
\hline Estimated population your hospital serves & & & \multicolumn{2}{|c|}{ Total number of telemedicine visits performed } & \\
\hline$<100,000$ & 46 & $9.6 \%$ & $0-10$ & 57 & $24.2 \%$ \\
\hline $100,000-500,000$ & 118 & $24.7 \%$ & $11-25$ & 75 & $31.8 \%$ \\
\hline $500,000-1,000,000$ & 100 & $21.0 \%$ & $25-50$ & 52 & $22.0 \%$ \\
\hline $1,000,000-2,000,000$ & 67 & $14.0 \%$ & $51-100$ & 27 & $11.4 \%$ \\
\hline$>2,000,000$ & 146 & $30.6 \%$ & $100+$ & 25 & $10.6 \%$ \\
\hline Hospital community & & & Trust in telemedicine anchor $\mathrm{q}$ & & \\
\hline Urban & 408 & $85.4 \%$ & Agree & 356 & $74.9 \%$ \\
\hline Suburban & 63 & $13.2 \%$ & Neutral or disagree & 100 & $21.1 \%$ \\
\hline Rural & 7 & $1.5 \%$ & & & \\
\hline \multicolumn{6}{|l|}{ Practice type } \\
\hline Academic/university hospital & 164 & $34.0 \%$ & & & \\
\hline "Privademic" (academic/private combined) & 128 & $26.6 \%$ & & & \\
\hline Private group, $<10$ practitioners & 58 & $12.0 \%$ & & & \\
\hline Private group, $>10$ practitioners & 20 & $4.1 \%$ & & & \\
\hline Individual practice & 35 & $7.3 \%$ & & & \\
\hline Government/military hospital & 34 & $7.1 \%$ & & & \\
\hline Hospital employee & 29 & $6.0 \%$ & & & \\
\hline Other & 14 & $2.9 \%$ & & & \\
\hline
\end{tabular}

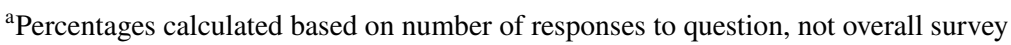

${ }^{b}$ Anchor question: "If you or a family member were a patient, do you believe the initial visit can be performed through telemedicine?" 
reviewing and explaining imaging (72.3\%), and formulating and communicating a treatment plan (67.7\%). Respondents had significantly less confidence in the physical exam portions of the evaluation; telemedicine was worse or much worse in assessing neurologic deficits (94.6\%), provocative testing (89.5\%) and myelopathy (89.0\%) (Fig. 2 and Table 2).

\section{Region}

In almost all analyses, region was not associated with differences in participant confidence in telemedicine (Figs. 1, 2). The significant differences noted in univariate analysis were all small and not significant after multivariate adjustment.

\section{Age}

On univariate analyses, participant age was not found to be associated with confidence in the telemedicine evaluation (Table 3). However, multivariate adjustment demonstrated that participants $>55$ years old were more likely to believe telemedicine was equivalent or better to in-person visits for the assessment of spinal deformity (OR: $2.51,95 \% \mathrm{CI}$ : 1.02-6.15) (Table 5).

\section{Telemedicine Platform}

Compared with telephone (audio only), the use of videoconferencing technology was associated with increased confidence in the ability of telemedicine to formulate and communicate a treatment plan when compared to in-person visits $(-0.08 \pm 0.69$ vs. $-0.41 \pm 0.68, p<0.001)$ (Table 3 ). This relationship was sustained in the multivariate model (OR: 3.88, 95\% CI: 1.71-8.84) (Table 5).

\section{Provider experience}

Providers who had performed $>50$ telemedicine visits demonstrated increased confidence in the ability of telemedicine to formulate and communicate a treatment plan $(0.02 \pm 0.80$ vs. $-0.32 \pm 0.69, p=0.018)$ and make an accurate diagnosis $(-0.63 \pm 0.88$ vs. $-0.88 \pm 0.69, p=0.049)$ (Table 3 ). On multivariate analysis, respondents experienced with telemedicine were more likely to believe telemedicine was equivalent or better than in-person visits in the ability to make an accurate diagnosis (OR: 2.37, 95\% CI: 1.03-5.43).

\section{Specialty}

Univariate analyses did not support a difference between orthopedic and neurosurgery in participant confidence in telemedicine evaluation. However, those with neurosurgery training described increased confidence in telemedicine for imaging review and explanation (OR: 3.67, 95\% CI: 1.39-9.71), compared to their orthopedic-trained colleagues.

\section{Provider trust in telemedicine}

Our anchor question for provider trust in telemedicine revealed that most respondents $(74.9 \%)$ believed that the initial visit could be performed through telemedicine. An affirmative response to the anchor question was associated with slightly increased confidence in telemedicine in almost all facets of the patient evaluation, with the exception of the assessment of myelopathy and imaging review (Table 4). However, none of these relationships were sustained in the multivariate analyses (Table 5).

\section{Discussion}

Our global survey of 485 spine surgeons demonstrates that the use of telemedicine in spine surgery is still in the early stages, with most participants having conducted relatively few telehealth visits at the time of the survey (Table 1). Overall, there remains considerable skepticism. Specifically, compared to in-person visits, spine surgeons generally felt

Table 2 Equivalency of telemedicine evaluation and entire sample

\begin{tabular}{|c|c|c|c|c|c|c|}
\hline & $N$ respondents & Much worse & Slightly worse & Equivalent & Slightly better & Much better \\
\hline \multicolumn{7}{|c|}{ How does telemedicine compare to in-person visits for the ability to... } \\
\hline Take a patient history & 221 & $4.5 \%$ & $31.2 \%$ & $56.1 \%$ & $4.1 \%$ & $4.1 \%$ \\
\hline Localize pain & 219 & $19.2 \%$ & $54.3 \%$ & $24.7 \%$ & $1.4 \%$ & $0.5 \%$ \\
\hline Assess neurologic deficits & 221 & $57.9 \%$ & $36.7 \%$ & $2.3 \%$ & $1.8 \%$ & $1.4 \%$ \\
\hline Assess myelopathy & 218 & $52.3 \%$ & $36.7 \%$ & $9.2 \%$ & $0.9 \%$ & $0.9 \%$ \\
\hline Assess spinal deformity & 220 & $27.3 \%$ & $45.5 \%$ & $24.5 \%$ & $1.4 \%$ & $1.4 \%$ \\
\hline $\begin{array}{l}\text { Perform provocative tests (straight leg raise, } \\
\text { Spurling's, Lhermitte's) }\end{array}$ & 220 & $50.9 \%$ & $38.6 \%$ & $8.6 \%$ & $0.9 \%$ & $0.9 \%$ \\
\hline Review imaging and explain patients & 220 & $5.0 \%$ & $22.7 \%$ & $54.1 \%$ & $13.6 \%$ & $4.5 \%$ \\
\hline Make an accurate diagnosis & 220 & $15.9 \%$ & $54.1 \%$ & $26.8 \%$ & $2.7 \%$ & $0.5 \%$ \\
\hline Formulate and communicate a treatment plan & 220 & $3.6 \%$ & $28.6 \%$ & $57.7 \%$ & $8.2 \%$ & $1.8 \%$ \\
\hline
\end{tabular}




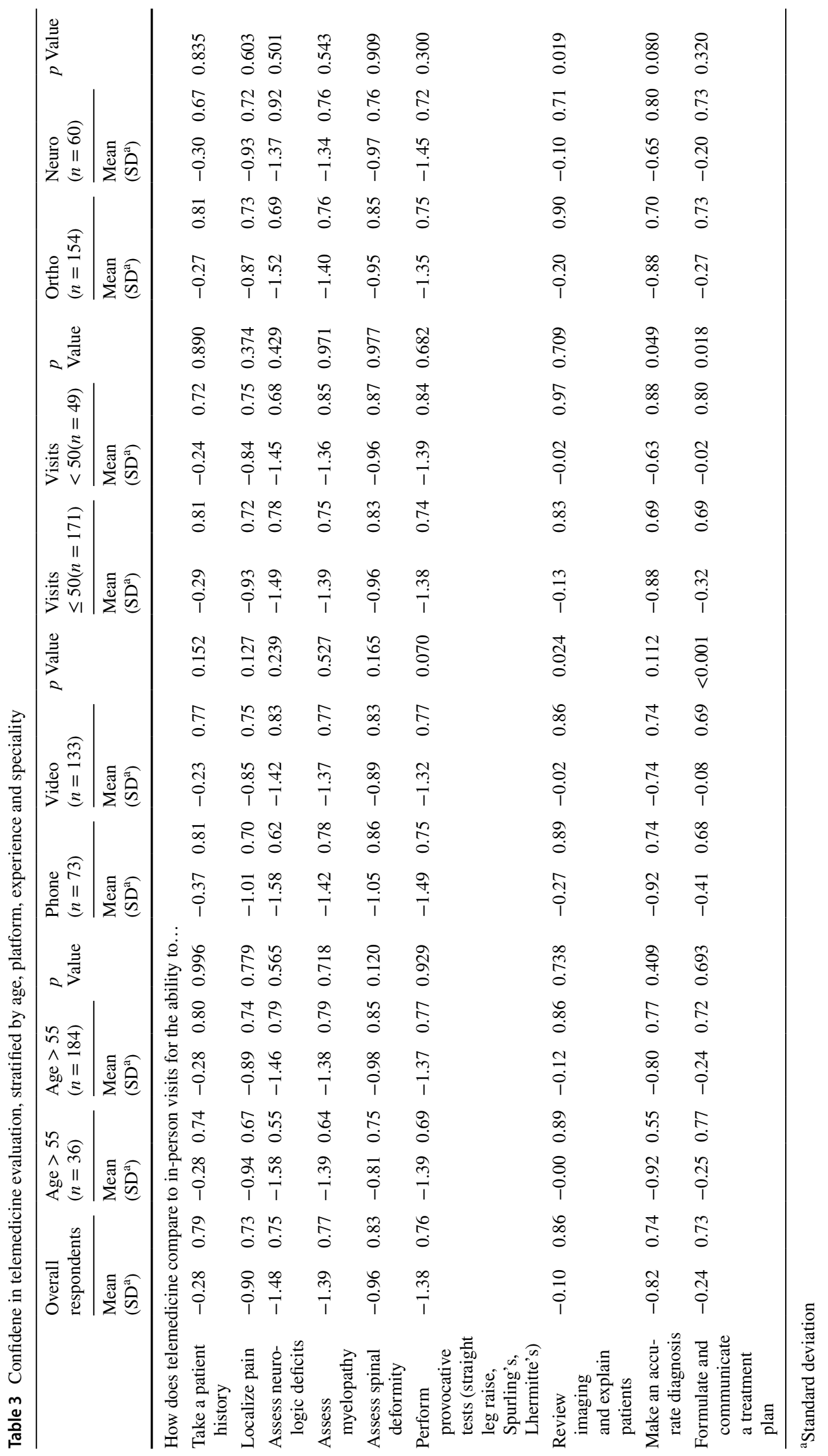


Table 4 Confidence in telemedicine evaluation, stratified by provider trust in telemedicine

\begin{tabular}{|c|c|c|c|c|c|}
\hline & \multicolumn{2}{|c|}{$\begin{array}{l}\text { Initial visit can be } \\
\text { done through tel- } \\
\text { emedicine }(n=123)\end{array}$} & \multicolumn{2}{|c|}{$\begin{array}{l}\text { No initial visit } \\
\text { through telemedicine } \\
(n=96)\end{array}$} & \multirow[t]{2}{*}{$p$ value } \\
\hline & \multicolumn{2}{|c|}{ Mean $\left(\mathrm{SD}^{\mathrm{a}}\right)$} & \multicolumn{2}{|c|}{ Mean $\left(\mathrm{SD}^{\mathrm{a}}\right)$} & \\
\hline \multicolumn{6}{|c|}{$\begin{array}{l}\text { How does telemedicine compare to in-person visits for the ability to... } \\
\text { ( }-2 \text { Telemedicine much worse, }-1 \text { slightly worse, } 0 \text { equivalent, } 1 \text { slightly better, } 2 \text { much better) }\end{array}$} \\
\hline Take a patient history & -0.12 & 0.81 & -0.49 & 0.73 & 0.002 \\
\hline Localize pain & -0.80 & 0.72 & -1.04 & 0.80 & 0.010 \\
\hline Assess neurologic deficits & -1.41 & 0.71 & -1.57 & $2.3 \%$ & 0.004 \\
\hline Assess myelopathy & -1.33 & 0.79 & -1.46 & 0.74 & 0.144 \\
\hline Assess spinal deformity & -0.76 & 0.83 & -1.20 & 0.78 & $<0.001$ \\
\hline Perform provocative tests & -1.26 & 0.81 & -1.53 & 0.66 & 0.007 \\
\hline Review imaging and explain to patients & -0.01 & 0.89 & -0.20 & 0.82 & 0.090 \\
\hline Make an accurate diagnosis & -0.67 & 0.67 & -1.02 & 0.78 & $<0.001$ \\
\hline Formulate and communicate a treatment plan & -0.10 & 0.70 & -0.42 & 0.74 & 0.003 \\
\hline
\end{tabular}

${ }^{\text {a }}$ Standard deviation

Table 5 Independent predictors of confidence in telemedicine evaluation

\begin{tabular}{|c|c|c|c|c|c|}
\hline Outcome of interest for multivariate model & Significant covariates & Odds ratio & $\begin{array}{l}95 \% \text { Confidence } \\
\text { interval }\end{array}$ & $p$ value & $\begin{array}{l}\text { Hosmer- } \\
\text { Leme- } \\
\text { show }\end{array}$ \\
\hline \multicolumn{6}{|l|}{$\begin{array}{l}\text { Compared to in-person visits, telemedicine is } \\
\text { equivalent or better to... }\end{array}$} \\
\hline Take a patient history & None & & & & 0.85 \\
\hline Localize pain & None & & & & 0.38 \\
\hline Assess neurologic deficits* &.- & & & & \\
\hline Assess myelopathy & None & & & & 0.13 \\
\hline Assess spinal deformity & Age $>55$ & 2.51 & $1.02-6.15$ & 0.045 & 0.85 \\
\hline Perform provocative tests & None & & & & 0.74 \\
\hline Review imaging and explain patients & Neurosurgery & 3.67 & $1.39-9.71$ & 0.009 & 0.59 \\
\hline Make an accurate diagnosis & Visits $>50$ & 2.37 & $1.03-5.43$ & 0.042 & 0.32 \\
\hline Formulate and communicate a treatment plan & Video & 3.88 & $1.71-8.84$ & 0.001 & 0.91 \\
\hline
\end{tabular}

Multivariate models controlled for age ( $>55$ vs. $\leq 55$ ), platform (video vs. phone), provider experience ( $>50$ vs. $\leq 50$ visits), region, specialty (orthopedic vs. neurosurgery, trauma/peds/other excluded), type of practice (academic/military/hospital employee vs. private/privademic), size of hospital's community, and type of hospital community. *Multivariate model not assessed given $<10$ events per variable

that telemedicine was inferior for every aspect of the patient evaluation (Fig. 1). However, there was a clear divergence between communication- and examination-based aspects of the patient evaluation.

The majority of survey respondents felt that telemedicine was at least equivalent to in-person visits for taking a patient history, reviewing and explaining imaging, and formulating and communicating a treatment plan (Fig. 2, Table 2). Although telemedicine remains in the early stages of adoption, these data are largely in keeping with the existing literature [10-13]. Agha et al. [12] conducted a non-inferiority, randomized controlled trial (RCT) of 221 patients seen in a medical office. They used a validated questionnaire to demonstrate that patient-centered communication tasks and clinical competence were perceived equally regardless of visit type (in-person or telemedicine). On the physician side,
Buvik et al. [13] surveyed orthopedic surgeons and found that provider assessment of communication was nearly identical between remote and in-person orthopedic consultations.

Importantly, both of these studies used videoconferencing. Additionally, because these studies were conducted by early adopters of telemedicine, it is likely that these providers were more experienced with telemedicine. These factors (the use of video and prior experience) were independently associated with increased confidence in telemedicine in our survey (Table 4).

Taken together, these data suggest that (when feasible) video conferencing should be the recommended platform for telemedicine visits. Additionally, as institutions integrate telemedicine into their practices, it will be important to provide exposure to this new technology in a graduated fashion. When possible, institutional adoption of telemedicine should include training on the differences between telemedicine and 


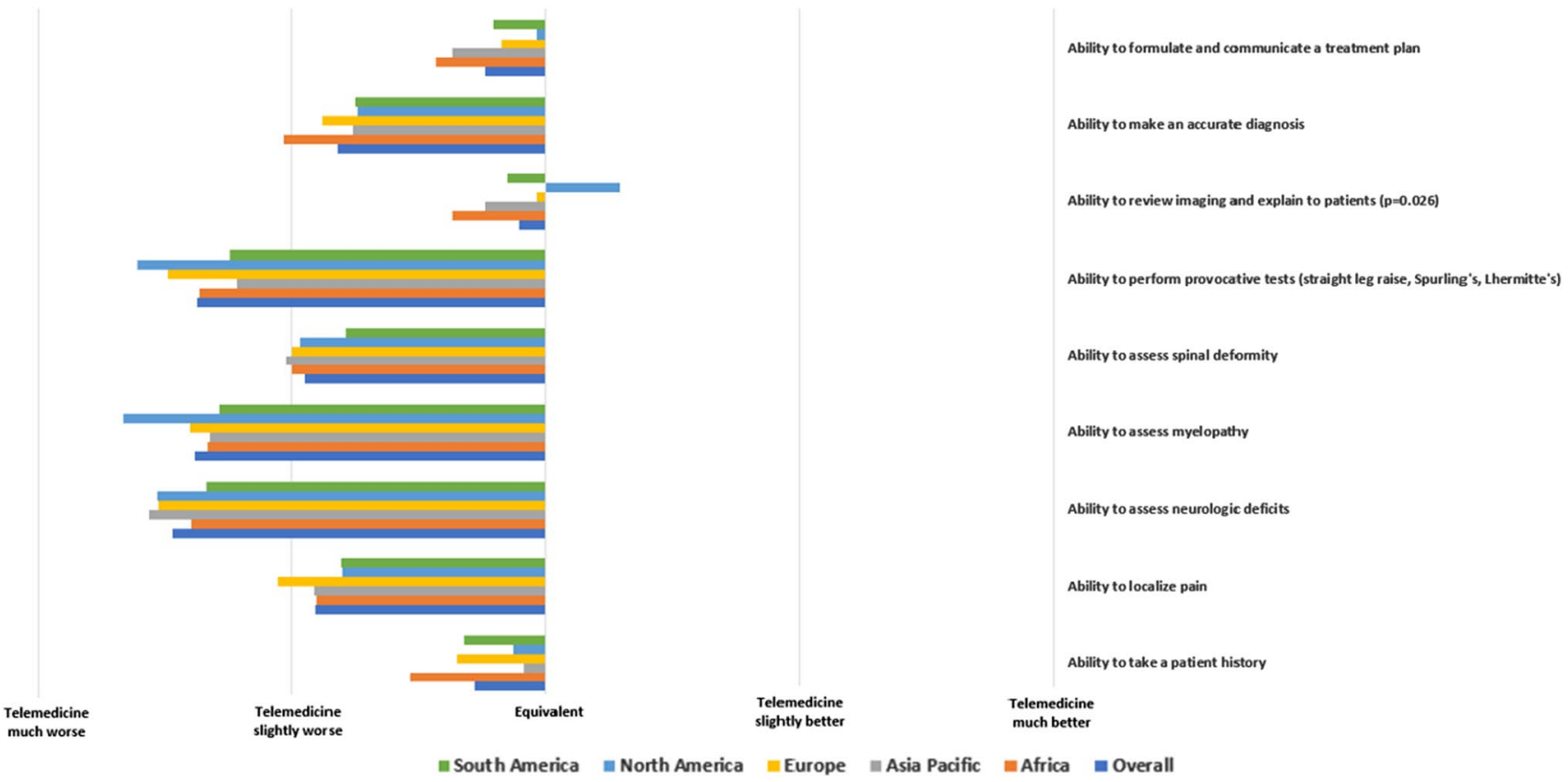

Fig. 1 Provider confidence in various patient evaluation tasks, stratified by region. There were no significant differences in responses by region ( $p>0.05$ for all comparisons)

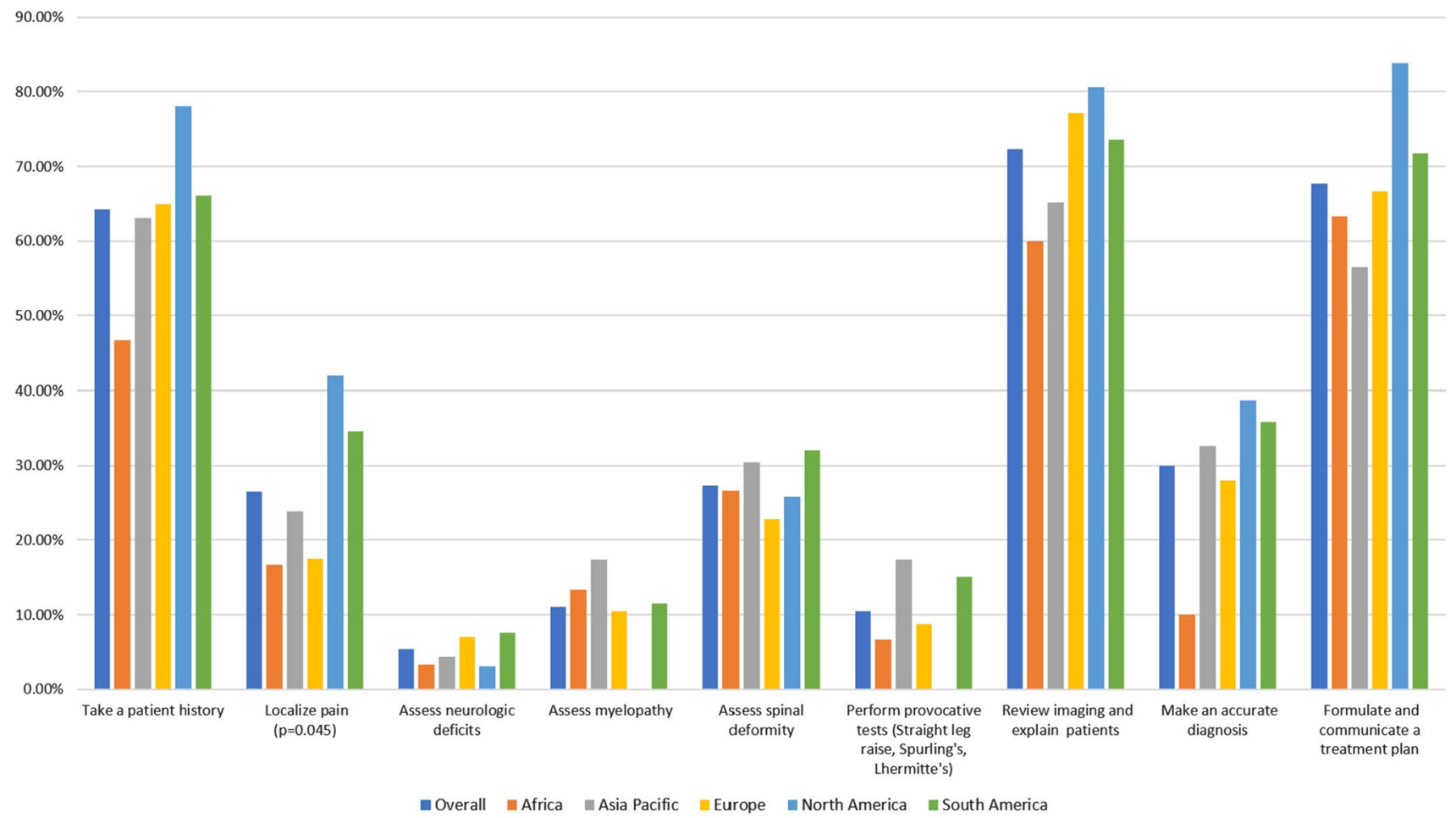

Fig. 2 Equivalency of telemedicine for patient evaluations, stratified by region. The only significant difference was noted in the equivalency of telemedicine to localize pain ( $p=0.045, p>0.05$ for all other comparisons)

in-person visits, simulated visits, exposure of house staff to telemedicine and accounting for an "adjustment phase" for new providers $[6,7,16]$, similar to the steps taken when onboarding a new provider to the practice.
In contrast to communication-based tasks, surgeon confidence in physical-exam-based patient evaluation (e.g., assessment of myelopathy, neurologic deficits, and provocative testing) was universally low. Almost all physicians 
( $90 \%)$ considered telemedicine slightly to be much worse than in-person visits for these tasks.

The inability of telemedicine to replicate the hands-on physical exam is probably the largest limitation to its adoption [8]. Interestingly, the need for "advanced physical exam maneuvers" has even been employed as an exclusion criterion from participation in orthopedic telemedicine RCTs [13]. Several authors have suggested strategies to improve the remote physical exam: asking patients to use weights (or body-weight maneuvers) and self-rate the required effort $[6$, 14], using composite movements [15] and utilizing technology to make reproducible measurements [16].

While our survey results leave little doubt about the telemedicine physical exam, it is certainly possible that a good patient history, imaging review, and a limited physical exam might be sufficient to make an accurate initial diagnosis in some patients [17]. In the situation where the diagnosis is not clear or surgical intervention is warranted, the provider may consider a follow-up in-person evaluation to supplement the telemedicine encounter. Certainly, there appears to be a learning curve to this type of visit (Table 5). In this sense, we expect providers to become more facile with their own telemedicine-based indications. Just as with any "new" technology, appropriate use criteria will have to be created for telemedicine, and the COVID19 pandemic makes this task ever more urgent. Expert consensus and high-quality research in this area are essential.

Our analysis is not without limitations. While there were several strengths of our survey population (international cohort split between regions, wide age range and years of practice, multiple spine surgery specialties and levels of experience with telemedicine, etc.), we only made the survey available for two weeks, capturing only a small portion of AO Spine members as a whole (485 [12.7\%] of 3805) and all response items were optional. Thus, the population may have strong opinions about telemedicine which influenced their decision to participate. In addition, the participants were mainly from large, urban areas; these are populous regions that have traditionally not relied on telemedicine [18]. Furthermore, the timing of the survey (during COVID-19) must be considered when interpreting our findings. It is difficult to predict whether surgeon opinion on telemedicine will change once the pandemic is under better control, especially if prepandemic regulations are re-instituted [5]. Finally, the study was not powered to rule out associations; thus, negative findings should be interpreted cautiously.

\section{Conclusions}

In conclusion, our global survey demonstrated that spine surgeons were confident in the ability of telemedicine to communicate with patients, but were concerned about its capacity to accurately make physical exam-based diagnoses. Video technology was strongly associated with increased confidence and should be used when available. Provider experience allows for increased confidence in how and when to use telemedicine. Given the limitations in the telemedicine physical exam, further research is needed to standardize physical examination methods and develop appropriate use criteria for this new tool.

Acknowledgements The authors would like to extend their sincere gratitude to Kaija Kurki-Suonio and Fernando Kijel from AO Spine (Davos, Switzerland) for their assistance with circulating the survey to AO Spine members.

\section{Compliance with ethical standards}

Conflict of interest The authors have no financial or competing interests to disclose.

Open Access This article is licensed under a Creative Commons Attribution 4.0 International License, which permits use, sharing, adaptation, distribution and reproduction in any medium or format, as long as you give appropriate credit to the original author(s) and the source, provide a link to the Creative Commons licence, and indicate if changes were made. The images or other third party material in this article are included in the article's Creative Commons licence, unless indicated otherwise in a credit line to the material. If material is not included in the article's Creative Commons licence and your intended use is not permitted by statutory regulation or exceeds the permitted use, you will need to obtain permission directly from the copyright holder. To view a copy of this licence, visit http://creativecommons.org/licenses/by/4.0/.

\section{Appendix 1: Survey}

\section{Telemedicine \& the Spine Surgeon - Spine Surgeon Perspectives and Practices Worldwide}

Purpose: Given the need for social distancing with COVID19 , telemedicine services have expanded around the world. We define telemedicine in this survey as clinical care provided remotely through videoconferencing or telephone. This survey seeks to determine:

1. 1.The extent of spine surgeon adoption of telemedicine

2. Satisfaction with telemedicine

3. Comparison of telemedicine to in-person visits

4. The use of telemedicine in research and training

5. Variations in perspectives and practices worldwide

The survey will not take more than 5-10 min to complete.Information obtained from this survey will be kept strictly confidential. The identity of all survey participants will remain anonymous. The findings of this survey will be disseminated via social media, journals, and other media platforms.Deadline to respond is May 31, 2020.Thank you in advance for taking the time to fill out this survey. 


\section{Demographic data}

1. Country of Spine Practice (select one)

2. Region of Spine Practice
a) Africa
b) Asia
c) Australia
d) Europe
f) North America
g) South America

3. What is your gender?
a. Male

b. Female

4. Age
a. $25-34$
b. $35-44$
c. $45-54$
d. 55-64
e. 65 or older

5. How many years have you been practicing spine surgery?
a. $<5$
b. $5-10$
c. $10-15$
d. $15-20$
e. $20+$

6. What is your specialty?
a. Orthopedics
b. Neurosurgery
c. Trauma
d. Pediatric Surgery
e. Other (please specify)

7. What percent (\%) of your practice is (sum total should be $100 \%$ ):
a. Adult Deformity
b. Degenerative
c. Pediatric
d. Trauma/Spinal Cord Injury
e. Tumor
f. Infections

8. Practice Type
a. Academic/University affiliated
b. "Privademic" (Academic/Private combined)
c. Private group, $<10$ practitioners
d. Private group, $>10$ practitioners
e. Individual practice
f. Government/Military Hospital
g. Hospital Employee
h. Other (please specify)

9. How would you define your hospital community?
a. Urban
b. Suburban
c. Rural 
10. What is the approximate population your hospital's serves?
a. $<100,000$
b. $100,000-500,000$
c. $500,000-1,000,000$
d. $1,000,000-2,000,000$
e. $>2,000,000$

11. Approximately what percentage of your time at work do you devote to the following:
a. Research (Slider from $0-100 \%$ )
b. Clinical Care (Slider from $0-100 \%)$
c. Teaching (Slider from $0-100 \%$ )

\section{General thoughts on Telemedicine}

12. Over the past few weeks, have you seen patients via telemedicine?
a. Yes
b. No, we do not have telemedicine options available, but I am a believer in telemedicine
c. No, I am not using telemedicine. I do not think telemedicine give any advantage

13. Over the past few weeks, what percentage of your patients have you seen via telemedicine?

a. Percentage of patients: (Slider from $0-100 \%$ )

14. What is your main type of telemedicine platform that you use?
a. Secure EMR-Integrated System
b. Secure non-EMR-Integrated System
c. Non-secure (Facetime, Skype, etc.)
d. Phone Call (no video)
e. Other (please specify)
f. None

15. Enter the name of the type of telemedicine platform you used
a. I don't know
b. System/Platform Name:

16. The telemedicine platform was easy to use.
a. Strongly agree
b. Agree
c. Undecided
d. Disagree
e. Strongly Disagree

17. How many telemedicine visits have you done?
a) $<10$
b) $11-25$
c) $26-50$
d) $50-100$
e) $100+$

18. How has your opinion about telemedicine changed as you have done more visits?
a) better than it was
b) worse than it was
c) my opinion has not changed
Comments: 
19. How often do technical difficulties delay or disrupt the visit?
a. Often $(50 \%+)$
b. Frequently (30-50\%)
c. Sometimes (15-30\%)
d. Rarely (0-15\%)
e. Never $(0 \%)$

20. A telemedicine visit requires of my time than an in-person visit
a. More
b. The same amount
c. Less

21. Have you experienced any of the following challenges when delivering care via telemedicine? (select all that apply)
a. Lack of access to internet
b. Lack of access to computer/phone with camera
c. Lack of access to telephone
d. Lack of technological literacy needed to use telemedicine
e. Technology implementation and maintenance costs
f. Decreased ability to perform physical examinations
g. Possible increased medicolegal exposure
h. Lack of reimbursement parity compared to traditional visits
i. Unclear billing codes
j. Regulatory barriers
k. Other (please specify)

22. Have your patients experienced any of the following challenges when receiving care via telemedicine? (select all that apply)
a. Lack of patient access to computer/phone with camera
b. Lack of patient access to telephone
c. Lack of technological literacy needed to use telemedicine
d. Perceived lack of privacy
e. Concern over paying for care received over telemedicine
f. Other (please specify)

23. For your patients, how do you perceive the following statements:

-2 (strongly disagree) ------ -1 (disagree) ------ 0 (neutral) ------ 1 (agree) ------ 2 (strongly agree)
a. The patients I see via telemedicine are generally younger
b. Older patients have a difficult time using telemedicine platforms
c. Telemedicine options are not available to patients with lower income levels
d. Patients seen over telemedicine tend to have a higher level of education
e. Patients seen over telemedicine are less likely to be minorities
f. Telemedicine is not significantly impacted by patient demographic factors

24. For your practice, how do you perceive the following statements?

$$
\text { -2 (strongly disagree) ------ -1 (disagree) ------ } 0 \text { (neutral) ------ } 1 \text { (agree) ------ } 2 \text { (strongly agree) }
$$

a. Telemedicine increases patient satisfaction

b. Telemedicine increases patient convenience

c. Telemedicine increases provider convenience

d. Telemedicine increases access to care for rural/international patients (patients from long distance)

e. Telemedicine decreases overhead for providers

f. Telemedicine decreases societal cost-savings (travel expenses, decreased hospital transfers, etc)

25. Before COVID-19 and social distancing mandates, what percentage of your practice were telemedicine visits? (Rate each answer between 0-100\%)

a. New patient

b. Follow-up visits before surgery (eg, check-in after injection, review of new imaging, etc.)

c. Postoperative visits 
26. During COVID-19 and social distancing mandates, what percentage of your practice have been telemedicine visits? (Rate each answer between 0-100\%)

a. New patient

b. Follow-up visits before surgery (eg, check-in after injection, review of new imaging, etc.)

c. Postoperative visits

27. If you or a family member were a patient, how do you perceive the following statements: -2 (strongly disagree) ------ -1 (disagree) ------ 0 (neutral) ------ 1 (agree) ------ 2 (strongly agree)

I believe:
a. The initial appointment can be done through telemedicine
b. Imaging review can be done over telemedicine
c. Post-operative care can be done through telemedicine
d. Patients should be seen at least once in person before being scheduled for surgery
e. Patients should be seen at least once in person postoperatively
f. It is clear to me how to charge for telemedicine

\section{Comparison of Telemedicine to In-person Visits}

How does telemedicine compare to in-person visits for the: (Presented as a grid)

28. Ability to take a patient history

a. telemedicine much better

b. telemedicine slightly better

c. Equivalent

d. telemedicine slightly worse

e. telemedicine much worse

29. Ability to localize pain
a. telemedicine much better
b. telemedicine slightly better
c. Equivalent
d. telemedicine slightly worse
e. telemedicine much worse

30. Ability to assess neurologic deficits
a. telemedicine much better
b. telemedicine slightly better
c. Equivalent
d. telemedicine slightly worse
e. telemedicine much worse

31. Ability to assess myelopathy
a. telemedicine much better
b. telemedicine slightly better
c. Equivalent
d. telemedicine slightly worse
e. telemedicine much worse

32. Ability to assess spinal deformity
a. telemedicine much better
b. telemedicine slightly better
c. Equivalent
d. telemedicine slightly worse
e. telemedicine much worse

33. Ability to perform provocative tests (straight leg raise, Spurling's, Lhermitte's)
a. telemedicine much better
b. telemedicine slightly better
c. Equivalent
d. telemedicine slightly worse
e. telemedicine much worse 
34. Ability to review imaging and explain to patients
a. telemedicine much better
b. telemedicine slightly better
c. Equivalent
d. telemedicine slightly worse
e. telemedicine much worse

35. Ability to make an accurate diagnosis
a. telemedicine much better
b. telemedicine slightly better
c. Equivalent
d. telemedicine slightly worse
e. telemedicine much worse

36. Ability to formulate and communicate a treatment plan
a. telemedicine much better
b. telemedicine slightly better
c. Equivalent
d. telemedicine slightly worse
e. telemedicine much worse

37. For a telemedicine visit in which you request imaging for a patient, where does that test occur?
a. Same location as for in-person visit
b. Different location
c. The patient can choose the location

38. For a telemedicine visit in which you request a laboratory test for a patient, where does that test occur?

a. Same location as for in-person visit

b. Different location

c. The patient can choose the location

39. In general, how comfortable do you feel performing surgery after a telemedicine evaluation?

a. Extremely comfortable, I do not need to see most patients prior to surgery

b. Moderately comfortable, I request a short in-person visit prior to the day of surgery

c. Slightly comfortable, I prefer a formal new patient visit prior to surgery

d. Not at all comfortable, I do not indicate a patient for surgery over telemedicine

Telemedicine in Training/Research

40. Are trainees (residents, fellows, etc) present during your telemedicine visits with patients?
a. Yes

i. Approximately what percentage of your telemedicine visits are performed with a trainee present: (Slider 0-100\%)

b. No

c. I don't normally work with trainees

41. Are other doctors present during your telemedicine visits with patients?
a. Yes, other spine surgeons
b. Yes, other surgeons (not spine, i.e., approach surgeons)
c. Yes, the patient's primary care provider
d. No

42. Approximately what percentage of your telemedicine visits are performed with the following personnel? (Rate each answer between 0-100\%)

a. Other spine surgeon

b. Other surgeons (not spine, i.e., approach surgeons)

c. Patient's primary care provider 
43. Please provide your thoughts on the following questions as they relate to telemedicine and training:

-2 (strongly disagree) ------ -1 (disagree) ------ 0 (neutral) ------- 1 (agree) ------ 2 (strongly agree) -

---- Not applicable (I have not used telemedicine for trainees)

a. Telemedicine should be part of the medical school curriculum

b. Telemedicine should be incorporated as part of residency/fellowship training

c. Directed history taking can be taught via telemedicine

d. Interpretation of physical exam maneuvers can be taught via telemedicine

e. Interpretation of imaging studies can be taught via telemedicine

f. I prefer teaching via telemedicine to in person

g. Teaching over telemedicine is as effective as in person teaching

44. Do you think telemedicine should be part of a residency/fellowship candidate's training curriculum in the clinical setting?

a. Yes

b. No

45. Of the time spent by residency/fellowship candidates in the clinic setting, what percentage should be over:

a. Telemedicine: Slider (0-100\%)

b. In-person visits: Slider (0-100\%)

46. Which of the following research activities are you performing over telemedicine? (select all that apply)

a. Patient recruitment/enrollment (obtaining patient consent)

b. Follow up physical examination

c. Follow up Health-related quality of life (HRQOL) \& other survey questionnaires

d. Follow up radiographs/imaging

e. Discuss research findings with research participants

f. Study group meetings

g. Other (please specify):

\section{References}

1. (2020) U.S. Telemedicine Market Statistics 2019-2025: Share Forecasts, Trends \& Growth drivers. In: MarketWatch

2. Dinesen B, Nonnecke B, Lindeman D et al (2016) Personalized telehealth in the future: A global research agenda. J Med Internet Res**

3. Forbes RC, Solorzano CC, Concepcion BP (2020) Surgical telemedicine here to stay: more support from a randomized controlled trial on postoperative surgery visits. Am J Surg. https:// doi.org/10.1016/j.amjsurg.2020.03.033

4. Louie PK, Harada GK, McCarthy MH et al (2020) The impact of COVID-19 Pandemic on Spine Surgeons Worldwide. Glob Spine J 10:534-552. https://doi.org/10.1177/2192568220925783

5. Makhni M, Riew G, Sumathipala M (2020) Telemedicine in orthopaedic surgery. J Bone Jt Surg A 102:1109-1115. https:// doi.org/10.2106/JBJS.F.01426

6. Yoon JW, Welch R, Alamin T et al (2020) Remote virtual spinal evaluation in the Era of COVID-19. Int J Spine Surg 14:7057. https://doi.org/10.14444/7057

7. Franco D, Montenegro T, Gonzalez GA et al (2020) Telemedicine for the Spine Surgeon in the age of COVID-19: multicenter experiences of feasibility and implementation strategies. Glob Spine J. https://doi.org/10.1177/2192568220932168

8. Donnally CJ, Vaccaro AR, Schroeder GD, Divi SN (2020) Is evaluation with telemedicine sufficient before spine surgery? Clin Spine Surg 00:1-4. https://doi.org/10.1097/BSD.0000000000 001027
9. Okoli C, Pawlowski SD (2004) The Delphi method as a research tool: an example, design considerations and applications. Inf Manag 42:15-29. https://doi.org/10.1016/j.im.2003.11.002

10. Grandizio LC, Mettler AW, Caselli ME, Pavis EJ (2020) Telemedicine after upper extremity surgery: a prospective study of program implementation. J Hand Surg Am. https://doi.org/10.1016/j. jhsa.2020.06.002

11. Debono B, Bousquet P, Sabatier P et al (2016) Postoperative monitoring with a mobile application after ambulatory lumbar discectomy: an effective tool for spine surgeons. Eur Spine J. https ://doi.org/10.1007/s00586-016-4680-4

12. Agha Z, Schapira RM, Laud PW et al (2009) Patient satisfaction with physician-patient communication during telemedicine. Telemed e-Health. https://doi.org/10.1089/tmj.2009.0030

13. Buvik A, Bugge E, Knutsen G et al (2016) Quality of care for remote orthopaedic consultations using telemedicine: a randomised controlled trial. BMC Health Serv Res 16:1-11. https:// doi.org/10.1186/s12913-016-1717-7

14. Tanaka MJ, Oh LS, Martin SD, Berkson EM (2020) Telemedicine in the era of COVID-19: the virtual orthopaedic examination. J Bone Joint Surg A 102(12):e57. https://doi.org/10.2106/ JBJS.20.00609

15. Palacín-Marín F, Esteban-Moreno B, Olea N et al (2013) Agreement between telerehabilitation and face-to-face clinical outcome assessments for low back pain in primary care. Spine (Phila. Pa. 1976) 38:947-952

16. Wochatz M, Tilgner N, Mueller S et al (2019) Reliability and validity of the Kinect V2 for the assessment of lower extremity 
rehabilitation exercises. Gait Posture 70:330-335. https://doi. org/10.1016/j.gaitpost.2019.03.020

17. Olldashi F, Latifi R, Parsikia A et al (2019) Telemedicine for neurotrauma prevents unnecessary transfers: an update from a nationwide program in Albania and analysis of 590 patients. World Neurosurg 128:e340-e346. https://doi.org/10.1016/j. wneu.2019.04.150
18. Call VRA, Erickson LD, Dailey NK et al (2015) Attitudes toward telemedicine in urban, rural, and highly rural communities. Telemed e-Health. https://doi.org/10.1089/tmj.2014.0125

Publisher's Note Springer Nature remains neutral with regard to jurisdictional claims in published maps and institutional affiliations. 